\title{
Die Amerikanisierung der politischen Online- Kommunikation in der wahlkampffreien Zeit Fallstudie: Eine Facebook Profilanalyse des rumänischen Präsidenten Klaus Iohannis
}

The Americanization of Online Political Communication in the ElectionFree Period. Case study: A Facebook Profile Analysis of the Romanian President Klaus Iohannis

\section{Lecturer Veronica CÂMPIAN, PhD}

Faculty of Communication, Public Relations and Advertising

Babeș-Bolyai University, Cluj-Napoca, Romania

E-mail: campian@fspac.ro

\section{Brîndușa Maria CERGĂ}

Faculty of Communication, Public Relations and Advertising

Babeș-Bolyai University, Cluj-Napoca, Romania

E-mail: brindusa.maria.c@gmail.com

\begin{abstract}
The article "The Americanization of on-line political communication in the external election period; Case study: An analysis of the Facebook profile of the Romanian President Klaus Iohannis" highlights the peculiarities of the Americanization of political communication in general and creates the transition to adapting this model to social media in Romania, with particular focus on Facebook. The analysis revolves around electoral time with a focus on non-election campaign time, assuming that political actors must continue to be communicatively active in order to be present in the mind of the electorate. The article sets out to investigate Iohannis' Facebook media profile during the external election period, starting with the research questions, whether the characteristics of Americanization are noticeable in Facebook communication and whether the frequency of posts during non-election campaign time experiences a descending change. 685 Facebook posts will be analyzed between
\end{abstract}


November 21, 2014 and November 23, 2017. The research is based on a quantitative content analysis completed by a trend analysis.

Keywords: Political communication; Americanization; On-line political discourse; Facebook, Political chronemics.

\section{Theoretischer Hintergrund}

\subsection{Die Amerikanisierung der rumänischen politischen Kommunikation}

Amerikanische Modelle der politischen Kommunikation prägen die Entwicklung von Kommunikationsstrategien weltweit und werden oft als ein Vorbild von den Strategen der politischen Akteure betrachtet. Die Umsetzung dieser Strategien ist zu einem Phänomen geworden. Die Annahme der amerikanischen Herangehensweise ohne jede Veränderung könnte den Wünschen und Erwartungen eines osteuropäischen Volkes nicht entsprechen. Jedoch greift das Phänomen der Amerikanisierung in den Kommunikationsstrategien der rumänischen politischen Akteure ein, wobei seine Merkmale im politischen Umfeld immer häufiger zu erkennen sind.

Die Merkmale der amerikanisierten Kommunikation beeinflussen den Ablauf der kommunikativen Versuche im politischen Umfeld. Der Wunsch, das Publikum schneller und gezielt anzusprechen, führt zur Ausarbeitung neuer Annäherungsstrategien. Diese kommen als aggressiver, also stärker und persuasiver vor. Die Dynamik wird von der ständigen Entwicklung des Internets und des Webs 2.0 bestimmt. Das Internet spielt eine bedeutende Rolle in der Aktualisierung der Techniken und der Annahme des amerikanischen Modells (vgl. Beciu, 2011, 229233). Von diesem Hintergrund ist die Amerikanisierung der rumänischen politischen Kommunikation durch die existierenden Gegebenheiten der politischen Sphäre zu untersuchen und zu bewerten.

Der schnelle, dynamische Takt der Kommunikation (vgl. Toader, Grigorași \& Frunză, 2011: 88) prägt alle Vermittlungstypen der heutigen on-line Botschaften. Kommunikatoren sollen ihre Inhalte ständig an die Erwartungen der Empfänger anpassen. Die durchgehende Kommunikation wird heutzutage im politischen Raum zur Priorität, denn die Kandidaten sollen ständig am öffentlichen Diskurs mitwirken. In diesem Kontext sind soziale Netzwerke die beste Wahl für politische Akteure. Soziale Netzwerke wie Facebook stellen die Echtzeitkommunikation im Mittelpunkt, so dass der politische Akteur in einem ständigen Dialog mit dem potentiellen Wähler sein kann. So liest man oft die Beschwerden der Bürger auf den Facebook Seiten regierender Politiker.

Die "Geschichte des Kandidaten“ (Toader, Grigorași \& Frunză, 2011, 88) als thematischer Kern ist ein wichtiges Merkmal des amerikanischen Modells der 
politischen Kommunikation. Sie stellt einen persönlichen Bezug des Empfängers der politischen Botschaft zum politischen Akteur her. Die autobiographischen Geschichten und Strategien wie z.B. Storytelling liegen heutzutage im Trend aufgrund ihrer Relevanz für die Positionierung des politischen Akteurs im Kopf des potentiellen Wählers. Zukunftspläne lassen sich vom politischen Akteur durch die persönliche Lebenserfahrung motivieren, da die Schwierigkeiten, die er während des Lebens bewältigt hat, als Grundlage seiner politischen Tätigkeit betrachtet werden können. Auch Ereignisse aus dem Privatleben der Politiker sind für die Öffentlichkeit interessant. Skandalöse, exklusive Inhalte erscheinen täglich in der Presse und bilden eine sekundäre, dennoch bedeutende Dimension eines politischen Akteurs.

Besonders in den sozialen Netzwerken soll das Publikum in einer freundlichen, unterhaltsamen Kommunikationsweise angesprochen werden, da das Internet jüngere Zielgruppen anspricht. Aus diesem Grund sind vor allem interaktive Präsentationsformen mit kurzen schriftlichen Inhalten wünschenswert. $\mathrm{Zu}$ den erfolgreichsten Darstellungsformen zählt auch die interaktive Gestaltung der Inhalte: piktographische und bildhaftere oder audio-visuelle Formate.

Die „Wahlwerbung“ (Toader, Grigorași \& Frunză, 2011, 88) führt eine neue Perspektive über den politischen Kandidaten ein. Der politische Akteur soll von den potentiellen Wählern immer als eine positive Gestalt empfunden werden. Seine Handlungen und Stellungnahmen sollen den Erwartungen der Wählerschaft entsprechen. Die von ihm getroffenen Maßnahmen und unterstützten Initiativen sollen im Einklang mit den Werten und Normen des angesprochenen Publikums sein. In diesem Sinn sind die Transparenz der Ziele und die Wiederholbarkeit der gesandten Botschaften gute Strategien für eine positive Beziehung zum Publikum. Folglich basieren die Mitteilungen auf den Initiativen und Leistungen des politischen Akteurs. Für den Kommunikationsfluss ist die Strategie „Spin Doctor" (Beciu, 2011, 236) auch sehr wichtig.

Obwohl dieses Idiom kein etablierter Fachbegriff ist, erscheint es oft in der Fachliteratur. Laut Esser (2008) ist die Funktion des Spin Doctors das Erzeugen von Wahlbotschaften, die sich auf für den jeweiligen Politiker vorteilhafte Themen richten (vgl. Esser, 2008, 21). So versucht der politische Akteur dem Publikum alle öffentlichen Themen auf eine solche Art und Weise vorzustellen, dass sie seiner Agenda entsprechen. Zum Beispiel kann der Politiker während eines Interviews die Antworten über mögliche Probleme innerhalb seiner Partei in eine unterschiedliche Richtung lenken, um vor allem seine eigenen Leistungen hervorzuheben. Die Fragen werden nur teilweise beantwortet, die Lage in der Partei bleibt für den Interviewer weiter unklar, dennoch werden die gewünschten Informationen über die Leistungen des politischen Akteurs kommuniziert. 


\subsection{Amerikanisierte Kommunikationsmodelle in den sozialen Medien}

Um die Umsetzung der amerikanischen Strategien durch die rumänischen Social Media Kommunikationskanäle zu forschen, müssen zunächst die Merkmale der politischen Kommunikation in Betracht gezogen werden. Die sozialen Netzwerke zeichnen sich durch ständige Interaktion und laufende Verfügbarkeit der Informationen aus. Aus diesem Grund ist auch der Wunsch nach der Anwesenheit politischer Akteure in einem Unterhaltungsmedium nachvollziehbar. Um in den sozialen Netzwerken eine erfolgreiche Beziehung zum Publikum zu schaffen, versuchen politische Akteure die gewünschten Themen in einer freundlichen, unterhaltsamen Weise zu veröffentlichen.

Die Häufigkeit der Veröffentlichungen ist besonders wichtig. Da alle Internetnutzer täglich mit einer riesig großen Menge an Botschaften überhäuft werden, muss die Vermittlung kontinuierlich und kohärent sein. Die Echtzeit-Kommunikation wird somit zu einem Vorteil. Ebenso wichtig ist auch die ständige Verfügbarkeit der Botschaften, da jede interessante Mitteilung gespeichert werden kann. Dank der Fragmentierungsmöglichkeiten der sozialen Netzwerke besteht die Möglichkeit, die „Geschichte des Kandidaten“ (Toader, Grigorași \& Frunză, 2011, 88) dem gezielten Publikum zu liefern.

\subsection{Die Amerikanisierungsmerkmale der politischen Kommunikation auf Facebook}

Die politische Kommunikation auf Facebook entsteht aufgrund des Wunschs der politischen Akteure, ein breites Publikum anzusprechen und somit mehrere Zielgruppen zu erreichen. Dafür bereiten die politischen Akteure gezielte Botschaften vor, die für Facebook entsprechend gestaltet werden sollen. Sowohl PR Berater, als auch Social Media Experten versuchen, Richtlinien für eine gelungene Facebook-Kommunikation zu bestimmen. Laut des Kommunikationswissenschaftlers Momoc (2014) kann die Kommunikationsweise der politischen Akteure die Bürger entweder zur Politik näherbringen oder sie davon abschrecken. (vgl. Momoc, 2014, 26).

Im rumänischen Umfeld ist die Bedeutung der Kommunikation auf Facebook auch aus dieser Perspektive zu berücksichtigen. Da Facebook ein Medium für alle ist (vgl. Brandom, 2018, 1), gibt es viele Facebook-Nutzer. Somit werden Transparenz und der politische Dialog als Mittel der Einbeziehung der Menschen in die politische Sphäre sehr bedeutsam. Zusätzlich soll der ganze Vorgang auch durch eine ständige und effiziente Kommunikation unterstützt werden.

\subsection{Die Gestaltung der politischen Botschaften auf Facebook nach amerikanischem Modell}

Sowohl politische Akteure als auch Unternehmen verwenden Facebook, um dem Publikum die gewünschten Botschaften, in einer wirkungsvollen Weise zu liefern. 
Deshalb versuchen die Experten der On-line Kommunikation, die Struktur und die Regeln für gelungene und wirksame Botschaften auf Facebook aufzustellen.

Die erste Bedingung, die erfüllt werden muss, ist die Festlegung der richtigen Häufigkeit der Posts. Laut einer von der On-line Plattform des Brands Fast Company durchgeführten Studie liegt die ideale Häufigkeit der Posts bei 0,5 bis 1,75 Posts pro Tag (vgl. Lee, 2014, 1).

Die zweite Bedingung bezieht sich auf die Relevanz der erwähnten Themen für die anzusprechende Zielgruppe. Um relevante Botschaften zu senden, müssen politische Akteure die wichtigsten Alltagsthemen kennen und ansprechen. Laut einer Studie, die im Jahr 2017 von iSense Solution durchgeführt wurde, sind für die Rumänen Themen wie Gesundheit, Wirtschaft, Familie und Freizeitaktivitäten sowohl im Fernsehen als auch in den sozialen Netzwerken wichtig. Dieselbe Studie sagt voraus, dass in der Zukunft auch die Themen Wissenschaft und Technik an Bedeutung gewinnen werden.

Eine dritte Bedingung für eine gelungene Facebook-Kommunikation sind die audio-visuellen Elemente. Bilder und Videos sind im Format der Facebook Posts üblicherweise einbegriffen. Diese helfen dabei, eine interaktivere Beziehung zwischen dem Sender und dem Empfänger der Botschaften zu schaffen. Für eine möglichst große Reichweite der Posts sind Bilder und Videos ein bedeutender Faktor.

Weiterhin ist die Länge der Facebook Posts ein wichtiges Element. Da Facebook eine schnelle, dynamische Echtzeit-Kommunikation anbietet, können sich die Nutzer der Plattform einer riesigen Anzahl von Informationsquellen bedienen. Die Schnelligkeit, mit der die Informationen erscheinen und die ständig neuen Meldungen haben einen großen Einfluss auf die Gestaltung der Posts. Man soll nicht vergessen, dass Facebook primär für audio-visuelle Inhalte entwickelt wurde. Heutzutage sind auch textbasierte Inhalte auf Facebook populär, dennoch sollen diese schriftlichen Botschaften nicht sehr lang sein. Der Meinung des Journalisten Corcoran nach beträgt die ideale Länge der Facebook-Texte weniger als 400 Zeichen (vgl. Corcoran, 2016, 1).

\subsection{Die Chronemik der politischen Kommunikation}

Im Kommunikationsverfahren der politischen Akteure sind die fortdauernde Formulierung und Veröffentlichung der gewünschten politischen und persönlichen Botschaften besonders relevant. Die politische Kommunikation ist ein Verfahren, das ununterbrochen sein sollte. Deshalb sind sowohl der wahlkampffreie Zeitraum als auch die Wahlkampfzeit zu beachten.

\subsubsection{Die wahlkampffreie Zeit}

Die Chronemik der Periode außerhalb der Wahlkämpfe ist ein Zentralpunkt der Kommunikationsstrategie eines politischen Akteurs. Der Zeitraum zwischen 
zwei Wahlkampagnen wird grundlegend in vier Zeitspannen (Rus, 2006, 418-419) aufgeteilt:

- Der Zeitraum unmittelbar nach dem Wahlkampf bezeichnet die ersten sechs Monate nach der jüngsten Wahlkampagne.

- Die späte Nachwahlkampfzeit beginnt sechs Monate nach dem Ende der vorherigen Wahlkampagne.

- Die Zwischenzeit bestimmt die zwei Jahren zwischen zwei Wahlkampagnen.

- Der Vorwahlkampf läuft im Jahr der Neuwahlen.

Besonders in der Ära des Internets hat die Vertrautheit der Wählerschaft mit dem Gesicht eines Politikers eine große Bedeutung. Folglich ist es ratsam, die Häufigkeit der politischen Kommunikation im Zeitraum außerhalb des Wahlkampfs nicht drastisch zu senken. Die sogenannte empfohlene Häufigkeit der politischen Kommunikation setzt die Einhaltung der geplanten und kontinuierlichen Kommunikationsvermittlung voraus. Die in der Wahlkampfzeit angewendeten komplexen Kommunikationstechniken sollen auch in der wahlkampffreien Zeit aktiv eingesetzt werden. Deshalb bietet dieser Zeitraum eine hervorragende Möglichkeit für die Analyse der kommunikativen Versuche eines politischen Akteurs.

\section{Die Facebook Profilanalyse von Klaus Iohannis}

Im Netz zogen seine Beliebtheit auf Facebook und das Gewinnen der Präsidentschaftswahlen im Jahr 2014 die Aufmerksamkeit der Presse und der Wählerschaft auf den Präsidenten Klaus Iohannis. Die Strategie von Klaus Iohannis wurde als sehr gut eingeschätzt, sogar sein Facebook Profil wurde zum Thema des Artikels „Der beliebteste europäische Präsident auf Facebook“, der auf der On-line Plattform der Zeitung Ziar de Iasi veröffentlicht wurde. Das Profil von Iohannis hatte am 14. März 2015 mehr als 1500000 Likes von Facebook-Nutzern.

Das Facebook-Profil von Klaus Iohannis wird durch eine zweistufige Inhaltsanalyse unter die Lupe genommen, die sowohl quantitative als auch qualitative Daten betrachtet. Die 685 Facebook Posts wurden anhand von mehreren Kriterien analysiert. Außer der sprachlichen Gestaltung der Botschaften wurden auch andere Aspekte in Betracht gezogen, die sowohl auf den Inhalt, als auch auf die Länge und die Art der Posts zielen. Der quantitative Teil der Forschung basiert auf den Durchschnittsquoten und dem Vergleich zwischen den festgelegten Zahlenwerten. Die durchgeführte Tendenzanalyse betrifft die Kategorisierung der thematischen Kerne, die in den Posts von Klaus Iohannis entdeckt worden sind. Die Forschung gründet auf vorwiegend quantitativen Daten.

Um die praktischen Auswirkungen der amerikanischen Kommunikationsmodelle auf die rumänische politische Kommunikation zu ermitteln, ist die Analyse des Profils von Klaus Iohannis ein relevantes Verfahren. Die Analyse bezieht sich auf das Facebook-Profil des derzeitigen Präsidenten Rumäniens. Ziel der gestellten Forschungsfragen sind sowohl die Untersuchung der Amerikanisierung der 
rumänischen politischen Kommunikation, als auch die der Kommunikationsfrequenz auf Facebook.

Die zwei Forschungsfragen der Studie lauten:

1. Ist die politische Kommunikation auf Facebook amerikanisiert?

2. Gibt es Schwankungen in der Kommunikationsdichte in der wahlkampffreien Zeit?

Weiterhin wird auch eine unterstützende Forschungsfrage formuliert:

Kommen die Merkmale der amerikanisierten Kommunikation im Aufbau, also in der Gestaltung, dem Inhalt und den audio-visuellen Teilen der Posts vor?

Die Stichprobe für die Untersuchung umfasst 685 Facebook-Posts von Klaus Iohannis. Die analysierte Periode betrifft den Zeitraum zwischen dem 21. November 2014 und dem 23. November 2017. Der Anfang der Untersuchung ist der 21. November 2014, als das Verfassungsgericht sein Amt als Präsident bestätigt hat. Die Wahlkampagnenzeit gilt ab diesem Datum als offiziell beendet.

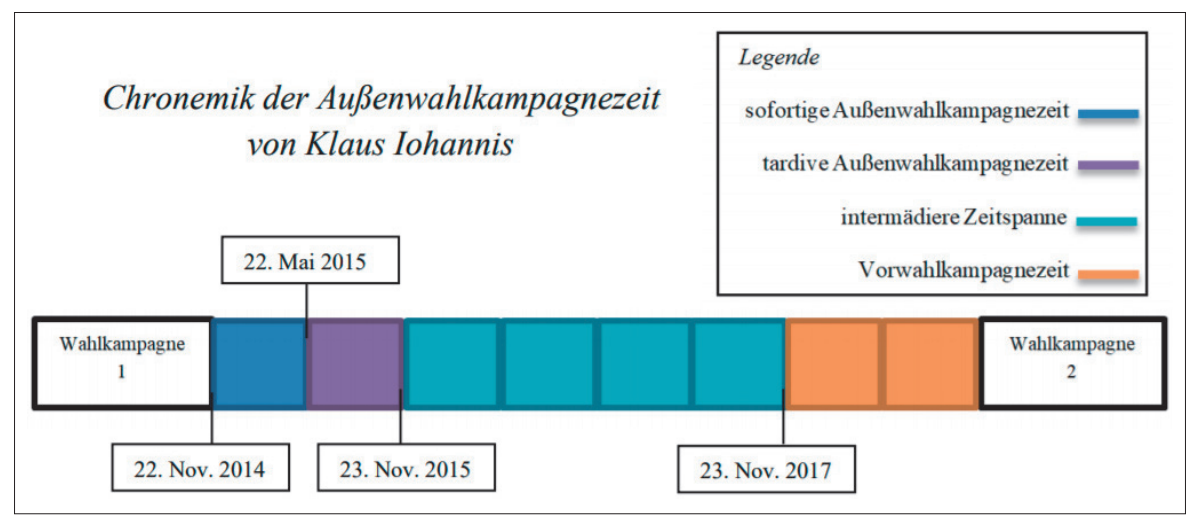

Abb. 1. Die Chronemik der wahlkampffreien Zeit von Klaus lohannis (eigene Darstellung)

\subsection{Die Art der Posts}

Das Facebook-Profil erlaubt dem Nutzer, selbsterstellte oder übernommene audio-visuelle Inhalte zu veröffentlichen, die mit oder ohne Text erscheinen. Da das soziale Netzwerk Facebook vor allem für Bilder geschaffen wurde, stehen diese im Mittelpunkt eines Posts. Trotzdem wird Facebook auch für schriftliche Mitteilungen sehr intensiv benutzt, indem die NutzerInnen ihre Meinungen und Bewertungen sehr oft durch Schrift äußern. Die vier möglichen Kategorien von mitgeteilten Inhalten auf Facebook sind Foto, Status, Video und Link.

Die Botschaften des Präsidenten bestehen aus einer Verknüpfung von Schrift und audio-visuellen Elementen. Von den untersuchten 685 Mitteilungen beinhalten die meisten Bilder oder Videos. In 60\% der Posts sind Bilder zu finden. 15\% der Meldungen, also 101 Posts, sind Videos. Insgesamt sind 75\% der geposteten 
Meldungen von audio-visuellen Inhalten geprägt. Nur 15 Posts veröffentlichen Links und bilden $2 \%$ der Gesamtanzahl an Meldungen (siehe Abb. 2). Man kann bemerken, dass ein bedeutender Teil der Posts durch Grafiken unterstützt wird. Aus der Gesamtanzahl der Mitteilungen sind 24 aus selbstständigen audio-visuellen Elementen gebildet und enthalten keine schriftliche Erklärung. Die potentiellen Wähler können somit Zugang zu den vom Präsidenten geposteten Bildern haben und können sich sogar Videos mit den Stellungnahmen von Iohannis anschauen.

Abb. 2. Die Art der Posts (eigene Darstellung)

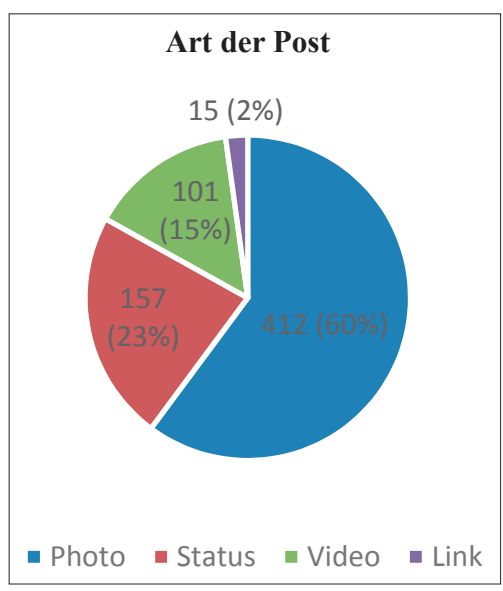

\subsection{Die Länge der Posts}

Ein Gesamtüberblick über die Art der Posts zeigt, dass 157 der Posts nur Text beinhalten. Die restlichen 528 sind dynamische Gebilde. Das Säulendiagramm (siehe Abb. 3) verdeutlicht die Länge der untersuchten Posts: die meisten Posts, 385, summieren zwischen 400 und 1000 Zeichen. Nur 23 Posts überschreiten 1000 Zeichen. Der einzige über 2000 Zeichen lange Post hat insgesamt 2.076 Zeichen und behandelt das Thema der Wahlen in der Republik Moldau.

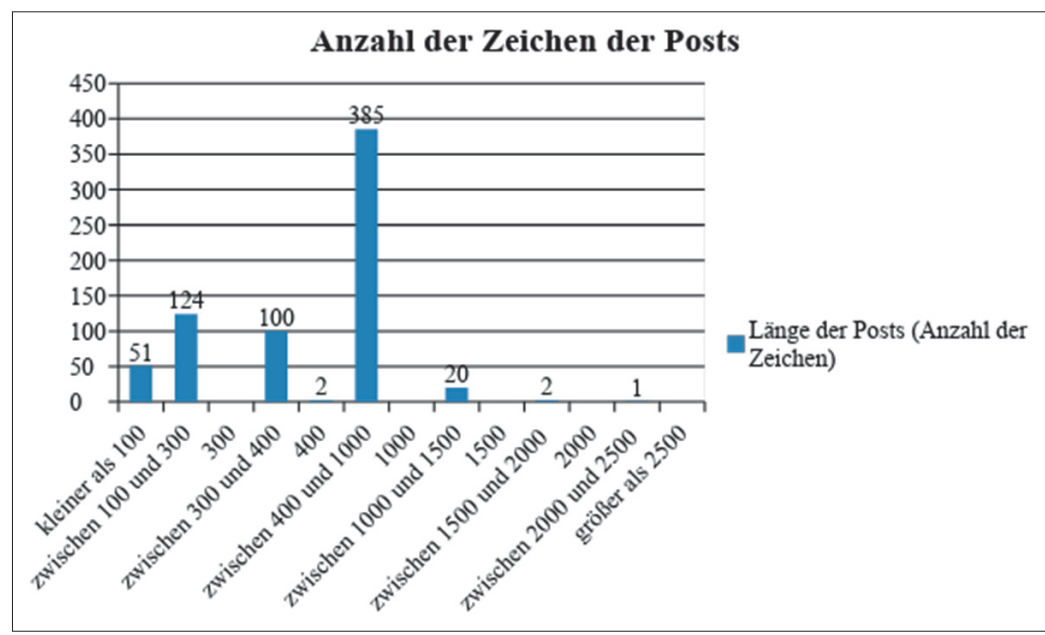

Abb. 3. Anzahl der Zeichen der Posts (eigene Darstellung)

277 Posts haben 400 Zeichen oder weniger: 2 Posts beinhalten genau 400 schriftliche Zeichen. Über 200 Meldungen weisen eine Struktur mit 100 bis 400 Zeichen 
auf und 51 Posts haben einen kurzen Text von weniger als 100 Zeichen. Die Länge der Texte beträgt im Durchschnitt 474 Zeichen.

\subsection{Die thematische Gestaltung der Posts}

Im Vergleich zu den negativ konnotierten Begriffen wie Angst, Bedrohung, Kritik, Reue, Traurigkeit oder Wut sind positive Ansichten und Stellungnahmen öfter in den Posts zu bemerken. Insgesamt wurden 116 Posts mit dem Indikator negativ und 24 mit dem Indikator neutral bewertet. Folglich sind die restlichen 545 Mitteilungen positive Stellungahmen des Präsidenten hinsichtlich der angesprochenen Themen.

Beispiele für negative Stellungnahmen in den Posts sind z.B.: „Was die Regierung letzte Nacht gemacht hat, ist inakzeptabel und ist eine Ordnungswidrigkeit. Der einzige Weg, den ich nicht akzeptiere, ist nichts dagegen zu tun. [...]"2 (1.02.2017) und „Ich verurteile auf Schärfste den bewaffneten Angriff, der heute Abend in München stattfand. Leider, unschuldige Menschen [...]"33 (22.07.2016). Als Beispiel für positive Stellungnahmen kann man folgende nennen: „Ich bin von dem Ausmaß der Demonstrationen sehr beeindruckt, die gestern Abend im ganzen Land stattgefunden haben. [...]"4 (12.07.2015) oder "Ich freue mich jedes Mal, wenn die Leistung rumänischer Schüler und Lehrer international anerkannt und geschätzt wird. Ich gratuliere den fünf Schülern und Lehrern [...] $]^{\prime 5}$ (25.07.2017). Auch neutrale Stellungnahmen sind in den Posts von Klaus Iohannis zu beobachten. Zum Beispiel: „Ich habe [...] den Ministerpräsidenten Dacian Ciolos, den Gouverneur der Nationalbank Rumäniens Mugur Isarescu und die Fraktionsvorsitzenden zu Konsultationen über die Ergebnisse des britischen Referendums eingeladen [...]"6 (24.06.2016).

Der "Spin Doctor" (Beciu 2011: 236) Strategie zufolge, sind die Mitteilungen auf einen positiven Diskurs gerichtet. Diese Blickrichtung ist für den politischen Akteur vorteilhaft, denn somit wird der Politiker der Wählerschaft näherge-

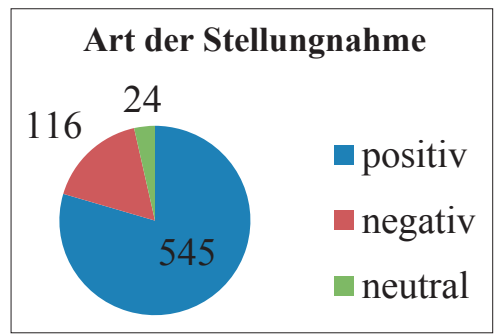

Abb. 4. Art der Stellungnahme bracht und zum Vertreter einer positiven Botschaft.

Es lässt sich weiterhin bemerken, dass die Kommunikationsstrategie von Klaus Iohannis auf Facebook thematische Schwerpunkte im Mittelpunkt hat. Die Mitteilungen bringen sowohl erfolgreiche Aspekte des Mandates von Iohannis als auch unterschiedliche Initiativen oder Leistungen des Präsidenten in den Vordergrund. Betrachtet man diese Eigenschaften der politischen Botschaften von Klaus Iohannis, wird die Verwendung der "Spin Doctor" (Beciu 2011: 236) Strategie in seiner Kommunikation offensichtlich. 


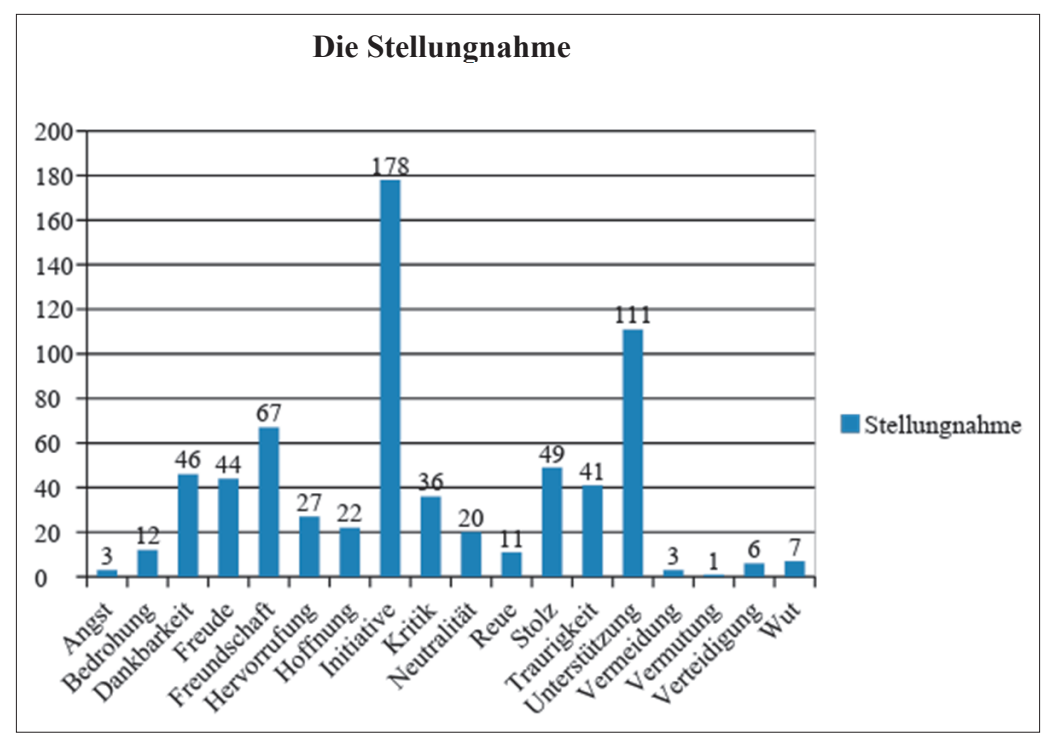

Abb. 5. Die Stellungnahme (eigene Darstellung)

Aus der Grafik (siehe Abb. 5) ist ersichtlich, dass der Indikator ,Initiative' die größte entsprechende Quote aller aufgezählten Indikatoren besitzt. 178 von den 685 Posts berichten über eine Initiative des Präsidenten und unterstreichen damit die Entscheidungskraft des Präsidenten. Diese Texte beinhalten sowohl imperative, herausfordernde Aussagen als auch konkrete Ziele und Pläne für bestimmte Situationen. Somit positioniert sich Klaus Iohannis als ein Präsident, der bereit ist, Entscheidungen zu treffen und angemessene amtliche Maßnahmen zu ergreifen. Seine Leistungen als Präsident bilden die Kategorie ,Aktivität'. Dazu gehören offizielle und formelle Aufgaben und Vorgaben, die vom Amt des Präsidenten verlangt werden.

\subsection{Die Häufigkeit der Posts}

Die Frage nach der Häufigkeit der Kommunikation in den sozialen Medien ist ein aktuelles Forschungsthema. Wie erwähnt, ist die Kohärenz der Kommunikation für das Image eines politischen Akteurs oder einer politischen Partei von groBer Bedeutung. Somit werden durch die Einhaltung der empfohlenen Häufigkeit der politischen Kommunikation die Kohärenz und Beständigkeit geschaffen.

Wie schon angesprochen, untersucht die vorliegende Analyse die Anzahl der Posts von Klaus Iohannis in der wahlkampffreien Zeit. Alle veröffentlichten Posts wurden gezählt und dem entsprechenden Erscheinungsmonat zugewiesen. Die Spitze des Liniendiagramms (siehe Abb. 6) entspricht dem Monat Januar 2015. In dieser Zeitspanne wurden 47 Post über wichtige aktuelle Ereignisse veröffentlicht. Damals stand er am Beginn seiner Amtszeit. Er blieb weiterhin sehr aktiv und 
wollte die Beziehung zu den Wählern pflegen. Klaus Iohannis hat zu diesem Augenblick eine intensive Öffentlichkeitsarbeit geleistet.

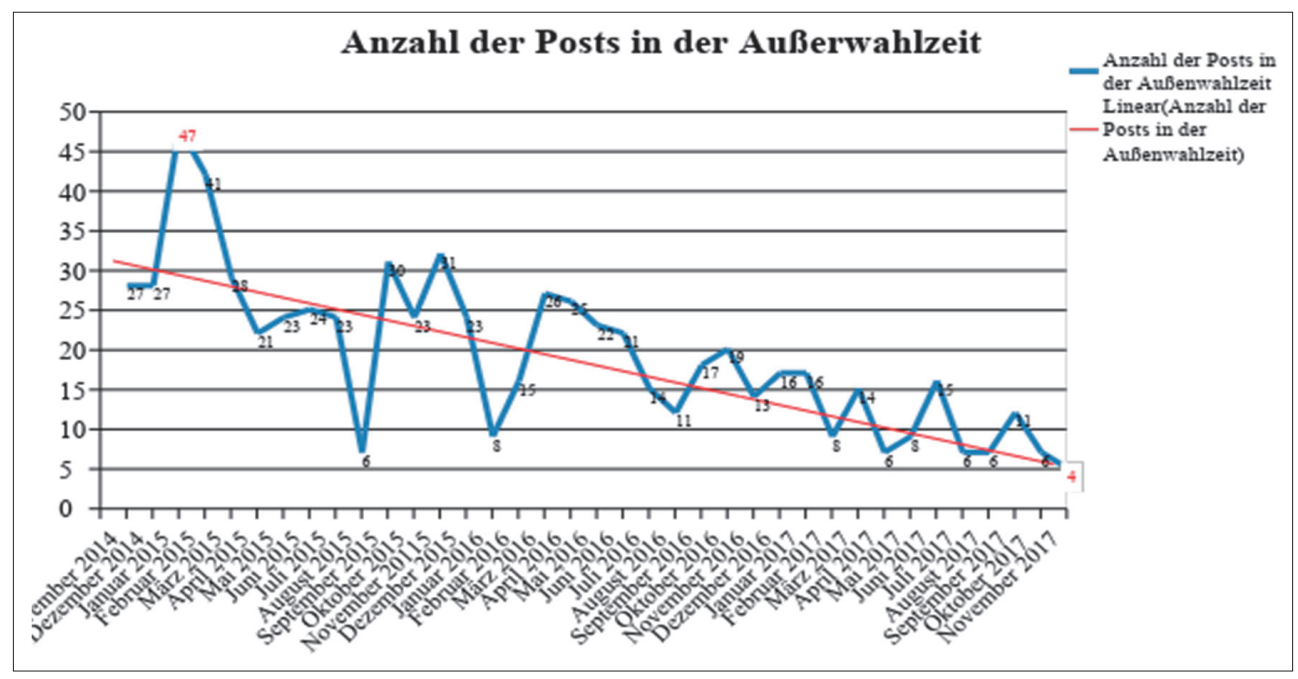

Abb. 6. Anzahl der Posts in der Außerwahlzeit (eigene Darstellung)

Dennoch ist eine senkende Tendenz sehr leicht zu beobachten. Mit der Zeit sinkt die Häufigkeitsquote der Posts. In den ersten Monaten, also Januar und Februar 2015 wurden 47 Posts, bzw. 41 Posts veröffentlicht. Die Anzahl von 30 Mitteilungen pro Monat wurde nur noch im September und November 2015 mit 30, bzw. 32 Posts überschritten. In allen anderen Monaten bleibt die Anzahl der Meldungen unter der Grenze von 30 Posts/Monat. Die Häufigkeit bleibt absteigend, so dass in den letzten neun Monaten maximal 15 Posts pro Monat mitgeteilt wurden.

Im August 2015, Januar 2016, Februar, April, Mai, Juli, August, Oktober und November 2017 hat der rumänische Präsident am wenigsten gepostet. Der Tiefpunkt wurde mit 4 Pots pro Monat im November 2017 erreicht. Die rote Tendenzlinie zeigt eine deutliche Senkung der Häufigkeit der Posts. Die Werte gehen graduell zurück und aus einem Durchschnitt von 30 Posts pro Monat wird ein Wert von ungefähr 5 Posts pro Monat erreicht.

Die größte Häufigkeit der Posts ist zwischen dem 21.11.2014 und dem 21.05.2015, also im Zeitraum unmittelbar nach dem Wahlkampf zu identifizieren. Dadurch kann das Medieninteresse im Online-Bereich für den Sieg von Iohannis erklärt werden. Die kleinste Veröffentlichungsquote wurde in den nächsten sechs Monaten, in der späten Nachwahlkapfzeit erreicht. Sie liegt dem niedrigsten Wert sehr nah, mit nur 0,75 Posts pro Tag. 
Tab. 1. Anzahl der Posts in der wahlkampffreien Zeit (eigene Darstellung)

\begin{tabular}{|c|c|c|c|}
\hline \multicolumn{4}{|c|}{ Anzahl der Posts in der wahlkampffreien Zeit } \\
\hline $\begin{array}{l}\text { Chronemik der wahl- } \\
\text { kampffreien Zeit }\end{array}$ & $\begin{array}{l}\text { Zeitraum unmittelbar nach } \\
\text { dem Wahlkampf }\end{array}$ & Späte Nachwahlkampfzeit & Zwischenzeit \\
\hline Zeitspanne & 21 Nov. 2014-21 Mai 2015 & 22 Mai 2015-22 Nov. 2015 & 23 Nov. $2015-23$ Nov. 2017 \\
\hline Anzahl Posts & 212 & 137 & 336 \\
\hline Durchschnitt Posts pro Tag & 1,18 & 0,75 & 1,03 \\
\hline & $0,5<1,18<1,75$ & $0,5<0,75<1,75$ & $0,5<1,03<1,75$ \\
\hline
\end{tabular}

Die Zwischenzeit beträgt eine Zeitspanne von 24 Monaten. Für den Gesamtüberblick muss, jedoch auch diese Zeitspanne bezüglich der Chronemik betrachtet werden. Um zu einem relevanten Endergebnis zu gelangen, soll der allgemeine Durchschnitt der Posts in der wahlkampffreien Zeit berechnet werden (siehe Tab. Nr. 2).

Tab. 2. Durschnitt der Posts in der wahlkampffreien Zeit (eigene Darstellung)

\begin{tabular}{|c|c|c|}
\hline \multirow{2}{*}{ Durchschnitt der Posts in der wahlkampffreien Zeit } & Durchschnitt Posts pro Monat & 18,51 \\
\cline { 2 - 3 } & Durchschnitt Posts pro Tag & 1,33 \\
\hline \multicolumn{2}{|c|}{$0,5<1,33<1,75$} \\
\hline
\end{tabular}

Die Resultate ergeben, dass in den drei Jahren, ungefähr 1,33 Posts pro Tag hochgeladen wurden. Monatlich wurden ungefähr 18,50 Mitteilungen auf Facebook gepostet. Daraus folgt, dass der Wert im Zwischenraum von 0,5 und 1,75 liegt.

\section{Schlussfolgerungen}

Die untersuchten Inhalte zeigen, dass die Amerikanisierung die politische Kommunikation nachweislich beeinflusst hat. Das Phänomen der Amerikanisierung ist eine Realität in der Sphäre der rumänischen politischen Kommunikation.

Die erste Forschungsfrage über die Amerikanisierung der politischen Kommunikation auf Facebook kann positiv beantwortet werden. Man kann schlussfolgern, dass die Merkmale der amerikanisierten Kommunikation sowohl im Aufbau als auch im Inhalt der Posts vorkommen. Die Analyse hat aber auch erwiesen, dass nicht alle Merkmale der Amerikanisierung in die Posts von Klaus Iohannis übernommen werden, so dass man nicht von einer vollständigen Amerikanisierung sprechen kann. Die rumänische politische Online Kommunikation ist also teilweise amerikanisiert.

Für diese Behauptung spricht auch, dass das Kommunikationsmodell nicht unverändert und gänzlich übernommen wurde, sondern dass die umzusetzenden Techniken an das existierende System angepasst wurden. 
Die Frage, ob Schwankungen in der Kommunikationsdichte in der wahlkampffreien Zeit zu registrieren sind, kann folgenderweise beantwortet werden: die Häufigkeit schwankt, die Veröffentlichungsquote der Posts sinkt nämlich in der wahlkampffreien Zeit. Man kann dieses Ergebnis damit erklären, dass sich die theoretischen Richtlinien für eine gelungene, kohärente und kontinuierliche Kommunikation in der Praxis nicht immer wiederfinden. Die fortlaufende Kommunikation wird als ein Erfolgsfaktor betrachtet. Trotzdem sind die Unterschiede zwischen der Kommunikation in der Wahlkampagnenzeit, wo die kommunikativen Strategien sehr stark gezielt und geplant werden und der Kommunikation in der wahlkampffreien Zeit deutlich. Klaus Iohannis hat immer wenigere Posts im Laufe der untersuchten Zeitspanne veröffentlicht.

Zusammenfassend ist zu bemerken, dass die politische Kommunikation amerikanisiert ist und, dass das positive Image eines politischen Akteurs nur durch eine kontinuierliche und kohärente Kommunikation aufrechterhalten werden kann. Die Ergebnisse der Analyse und die vorgestellten theoretischen Grundlagen bieten neue Perspektiven für die zukünftige Forschung im Bereich der politischen Kommunikation an. Die ständige technische Entwicklung und die große Bedeutung der Online Kommunikation in den sozialen Netzwerken führt zur Fragestellung, wie sich politische Akteure in der digitalisierten Öffentlichkeit präsentieren sollen und wie sie sich an die neuen Richtlinien der politischen Kommunikation im Online-Bereich am besten anpassen können.

\section{Literaturliste:}

1. Beciu, C. (2011). Sociologia Comunicării și a Spațiului Politic, Editura Polirom, Bucharest.

2. Brandom, R. (2018). Mark Zuckerberg istn't ruling out a paid version of Facebook. Von The Verge, www.theverge.com am June 16, 2018.

3. Corcoran, L. (2016). What's the Perfect Length of a Facebook Post?, www.newswhip. com am June 18, 2018.

4. Esser, F. (2008). Spin Doctor. In Donsbach, W. (Hrsg.). International Encyclopedia of Communication, X. Ausgabe, Blackwell Publishing, Oxford.

5. Lee, K. (2014). The Social Media Frequency Guide: How Often to Post to Facebook, Twitter, LinkedIn, and More. Von Fast Company, www.fastcompany.com am 18.05.2018.

6. Momoc, A. (2014). Comunicarea 2.0: New Media, participare și populism, Editura Adenium, Iași.

7. Rus, F.C. (2006). PR Politic. In Marga, A., Mureșan, M., Ștefan, M., Rus, F.C., Luțaș, M., Lazăr, D., Balaban, D.C., Chereji, C.R. \& Mureșan, I. Științe ale Comunicării. Note de curs II, Editura Accent, Cluj-Napoca.

8. Toader, F., Grigorași, C. \& Frunză, S. (2011). Politica User Friendly, Editura Tritonic, București.

9. Ziar de Iași (2015). Klaus Iohannis, cel mai iubit președinte european pe Facebook (www.ziaruldeiasi.ro) am June 2, 2018. 